\section{Análise do efeito idade-período-coorte na mortalidade por câncer colorretal no Estado do Rio de Janeiro, Brasil, no período 1980 a 2014}

\author{
Analysis of the age-period-cohort effect on \\ mortality from colorectal cancer in Rio de Janeiro \\ State, Brazil, from 1980 to 2014
}

Brenda Gasparini 1,2

Marcus Valadão 2

Adalberto Miranda-Filho 3

Cosme Marcelo Furtado Passos da Silva 1

\title{
Resumo
}

O objetivo deste trabalho foi estimar a contribuição do efeito da idade, do período e da coorte de nascimento na mortalidade por câncer colorretal. Foram analisados dados de óbitos pela neoplasia entre indivíduos com mais de 35 anos de idade do Estado do Rio de Janeiro, Brasil, extraídos do Sistema de Informações sobre Mortalidade (SIM) entre 1980 e 2014. As taxas de mortalidade foram calculadas por gênero e faixa etária. O efeito da idade, período e coorte de nascimento foi estimado pelo método que utiliza funções estimáveis: desvios, curvaturas e drift, na biblioteca Epi do software R. As taxas de mortalidade por câncer colorretal padronizadas foram 27,37/100 mil homens e 21,83/100 mil mulheres em 2014. Verificou-se aumento da mortalidade pela neoplasia entre 1980 e 2014, sendo as taxas de mortalidade entre homens superiores às das mulheres após a década de 1990. O efeito idade foi observado com o aumento das taxas e o envelhecimento. A análise das gerações mostrou o menor risco de óbito nas coortes mais antigas comparadas às mais recentes coortes, o que pode estar ligado à adoção do estilo de vida ocidental. Tal cenário aponta a relevância da implantação de estratégias de rastreamento visando ao diagnóstico e ao tratamento precoce de lesões precursoras da doença.

Neoplasias Colorretais; Efeito Idade; Efeito Período; Efeito de Coortes

\author{
Correspondência \\ B. Gasparini \\ Rua Equador 831, Rio de Janeiro, RJ 20220-410, Brasil. \\ bregaspa@hotmail.com \\ 1 Escola Nacional de Saúde Pública Sergio Arouca, Fundação \\ Oswaldo Cruz, Rio de janeiro, Brasil. \\ 2 Instituto Nacional de Câncer José Alencar Gomes da Silva, Rio \\ de Janeiro, Brasil. \\ 3 Section of Cancer Surveillance, International Agency for \\ Research on Cancer, Lyon, France.
}




\section{Introdução}

O câncer colorretal possui relevância epidemiológica no cenário mundial por se tratar da terceira neoplasia mais frequente nos homens e da segunda nas mulheres. A ocorrência do câncer colorretal é superior a 1,3 milhão casos novos por ano, levando a óbito mais da metade dos acometidos pela doença. A estimativa da incidência da neoplasia para o ano de 2012 foi de 746 mil casos novos em homens e 614 mil em mulheres (Ferlay J, Soerjomataram I, Ervik M, Dikshit R, Eser S, Mathers C, et al. GLOBOCAN 2012 v1.0. http://globocan.iarc.fr, acessado em 05/Out/2014). No Brasil, para o ano de 2016, estimaram-se novos casos em 16.660 homens e em 17.620 mulheres. Destacou-se o Rio de Janeiro como o segundo estado do país com a maior estimativa da incidência da neoplasia (4.660 novos casos em ambos os sexos), sendo superado apenas por São Paulo (Instituto Nacional de Câncer José Alencar Gomes da Silva. http://www.inca.gov.br/estimativa/2016/, acessado em 05/Nov/2015).

A incidência e a mortalidade por câncer colorretal variam amplamente no mundo em diferentes gradientes de acordo com o Índice de Desenvolvimento Humano (IDH) 1. Foram identificados três padrões de distribuição da doença: (i) elevação de ambas as taxas nas mais recentes décadas em países em rápida transição econômica, dentre eles o Brasil; (ii) aumento da incidência e diminuição da mortalidade em países com alto IDH, incluindo Canadá, Reino Unido, Cingapura e Dinamarca; e (iii) diminuição de ambas as taxas nos países com muito alto IDH como Estados Unidos, Japão e França 1. Certamente, mudanças na prevalência de fatores relacionados ao estilo de vida ligado à extensão da adoção de hábitos ocidentais são, em parte, responsáveis pela variação na incidência da neoplasia 1 . No Brasil, o padrão epidemiológico do câncer colorretal é pouco conhecido, contudo alguns estudos apontaram o aumento da mortalidade nos últimos anos 2,3,4,5.

A descrição da mortalidade por câncer colorretal em nível local é medida importante para avaliar o impacto da doença ao longo do tempo e, assim, revelar padrões que possam suscitar hipóteses a futuros estudos. Com objetivo de ampliar o escopo das análises de tendência em epidemiologia do câncer, foram desenvolvidos os modelos idade-período-coorte 6 . No entanto, é importante compreender os conceitos que englobam a modelagem, descritos previamente por Robertson \& Boyle 7. O efeito idade representa as mudanças das taxas associadas à idade. Os efeitos período e coorte representam as mudanças das taxas associadas ao tempo. No efeito período, as mudanças nas taxas ocorrem em razão de fatores localizados em um momento do tempo e que influenciam a todos os grupos etários simultaneamente. No efeito coorte, os fatores afetam uma geração e provocam mudanças nas taxas de magnitude diferente em sucessivos grupos etários e períodos.

O objetivo do presente estudo foi estimar a contribuição do efeito da idade, do período e da coorte de nascimento na mortalidade por câncer colorretal no Estado do Rio de Janeiro, Brasil.

\section{Materiais e métodos}

\section{Dados e população de estudo}

Inicialmente, foi realizado um estudo descritivo da mortalidade por câncer colorretal na população adulta com 35 anos ou mais, residentes no Estado do Rio de Janeiro, entre os anos de 1980 e 2014. A distribuição dos óbitos foi obtida mediante consulta ao Sistema de Informações sobre Mortalidade (SIM) do Ministério da Saúde, disponível na página de Internet do Departamento de Informática do Sistema Único de Saúde (DATASUS. http://www2.datasus.gov.br/DATASUS/index.php, acessado em 08/Ago/2017). No período destacado, duas revisões da Classificação Internacional de Doenças (CID) estiveram em vigor: a CID-9 até o ano de 1995 e a CID-10 de 1996 a 2014. Foram incluídos no estudo apenas os óbitos que tiveram como causa básica de óbito o câncer colorretal. Na CID-9, os códigos correspondentes à categoria de interesse (câncer de cólon e reto) foram: 153 e 154. Já na CID10, foram: C18 (neoplasia maligna do cólon), C19 (neoplasia maligna da junção retossigmoide) e C20 (neoplasia maligna do reto). As informações sobre a população foram obtidas de dados do Instituto Brasileiro de Geografia e Estatística (IBGE), disponibilizados pelo DATASUS (http://www2.datasus. gov.br/DATASUS/index.php?area=0206, acessado em 08/Ago/2017). 
Foram calculadas taxas de mortalidade bruta e específica por faixa etária e sexo para cada ano do estudo. Em seguida, realizou-se a padronização por idade das taxas pelo método direto que usa como padrão a população mundial proposta por Segi e modificada por Doll et al. 8 .

\section{Análises estatísticas}

Para construir os modelos de regressão idade-período-coorte, o número de óbitos e a população em risco foram agrupados em intervalos regulares, iniciando-se na faixa etária de 35-39 anos até 80 anos e mais (10 faixas etárias) e no período de 1980-1984 até 2000-2014 (7 períodos). As coortes de nascimento tiveram início em 1900 até 1975 (16 coortes). Com a base de dados tabelados, os efeitos da idade, do período e da coorte foram modelados como variáveis categóricas, gerando um grande número de parâmetros a ser estimado. Para limitar os parâmetros a um número razoável e obter curvas apropriadas para os efeitos estimados, as faixas etárias foram agrupadas em intervalos de 5 anos. Como o câncer é uma doença rara, o uso de intervalos ajuda a melhorar a estabilidade dos dados 9 .

O modelo para os dados tabelados foi escrito sob a forma de uma regressão log-linear tal como 10,11:

$$
\log \left(E\left(r_{i j k}\right)\right)=\log \left(\frac{\theta_{i j k}}{N_{i j k}}\right)=\mu+\alpha_{i}+\beta_{j}+\gamma_{k}
$$

onde $E\left[r_{\mathrm{ijk}}\right]$ é a taxa de mortalidade esperada em uma idade i, período j e coorte $\mathrm{k}$; $\theta_{\mathrm{ijk}}$ é o número esperado de óbitos que assume ter uma distribuição de Poisson; $\mathrm{N}_{\mathrm{ijk}}$ é a população sob risco de morte (sendo o $\log \left(\mathrm{N}_{\mathrm{ijk}}\right)$ o termo offset ou termo de ajustamento $\log$-linear para tabelas de contingência); $\mu$ representa a média do efeito ou intercepto; $\alpha_{i}$ é a i-ésima linha i do efeito idade para $i=1, \ldots, \alpha$ grupos etários; $\beta_{\mathrm{j}}$ é a j-ésima coluna do efeito período para $\mathrm{j}=1, \ldots, \mathrm{p}$ períodos; e $\gamma_{\mathrm{k}}$ é a k-ésima diagonal do efeito coorte para $\mathrm{k}=1, \ldots,(\alpha+\mathrm{p}-1)$ coortes. Nesse modelo apresentado, os três efeitos atuam de forma aditiva sobre o logaritmo da taxa.

A principal dificuldade no ajuste de um modelo envolvendo esses três fatores é a relação linear entre idade, período e coorte. Qualquer modelo que inclua as três variáveis sobre uma escala linear será limitado porque a coorte de nascimento corresponde à diferença entre o ano do óbito e a idade ao morrer, levando a uma condição conhecida como problema de não identificabilidade 10 . Não há consenso quanto à melhor metodologia para resolver tal problema. Este estudo optou por estimar os parâmetros do efeito idade-período-coorte pelas funções estimáveis: desvios, curvaturas e drifts 11,12 . Foram utilizados como referência a coorte de nascimento de 1935-1939 e o período de 1980-1984.

A estatística deviance foi utilizada para avaliar o ajuste do modelo. Trata-se de duas vezes o logaritmo da função de verossimilhança do modelo completo em relação ao logaritmo da função de verossimilhança do modelo estimado. A contribuição dos efeitos foi avaliada pela comparação da deviance do modelo estimado com o efeito específico em relação ao modelo completo (idade-período-coorte). Mudanças nessa estatística entre dois modelos assume uma distribuição $\chi^{2}$ com graus de liberdade iguais à diferença do número de parâmetros dos dois modelos ${ }^{12}$. Foram considerados estatisticamente significativos os valores de $\mathrm{p} \leq 0,05$.

Para as análises estatísticas foi utilizada a biblioteca Epi 2.0 (https://CRAN.R-project.org/ package=Epi) no software livre R versão 3.3.2 (The R Foundation for Statistical Computing, Viena, Áustria; http://www.r-project.org).

Este estudo foi aprovado pelo Comitê de Ética em Pesquisa da Escola Nacional de Saúde Pública Sergio Arouca, Fundação Oswaldo Cruz (CAAE no 51697215.6.0000.5240).

\section{Resultados}

No período entre 1980 e 2014, ocorreram 38.646 óbitos por câncer colorretal no Estado do Rio de Janeiro em indivíduos com 35 anos ou mais. Desses, $54,7 \%$ ocorreram em mulheres e 45,3\% em homens. Em 2014, a taxa de mortalidade padronizada foi 27,3/100 mil homens e 21,8/100 mil mulheres.

A Figura 1 apresenta o padrão temporal das taxas de mortalidade ajustadas, em homens e mulheres, no Rio de Janeiro, entre os anos de 1980 a 2014. Foi possível observar que as taxas de mortalidade 
Figura 1

Evolução da taxa padronizada de mortalidade por câncer colorretal de acordo com o sexo. Estado do Rio de Janeiro, Brasil, 1980 a 2014.

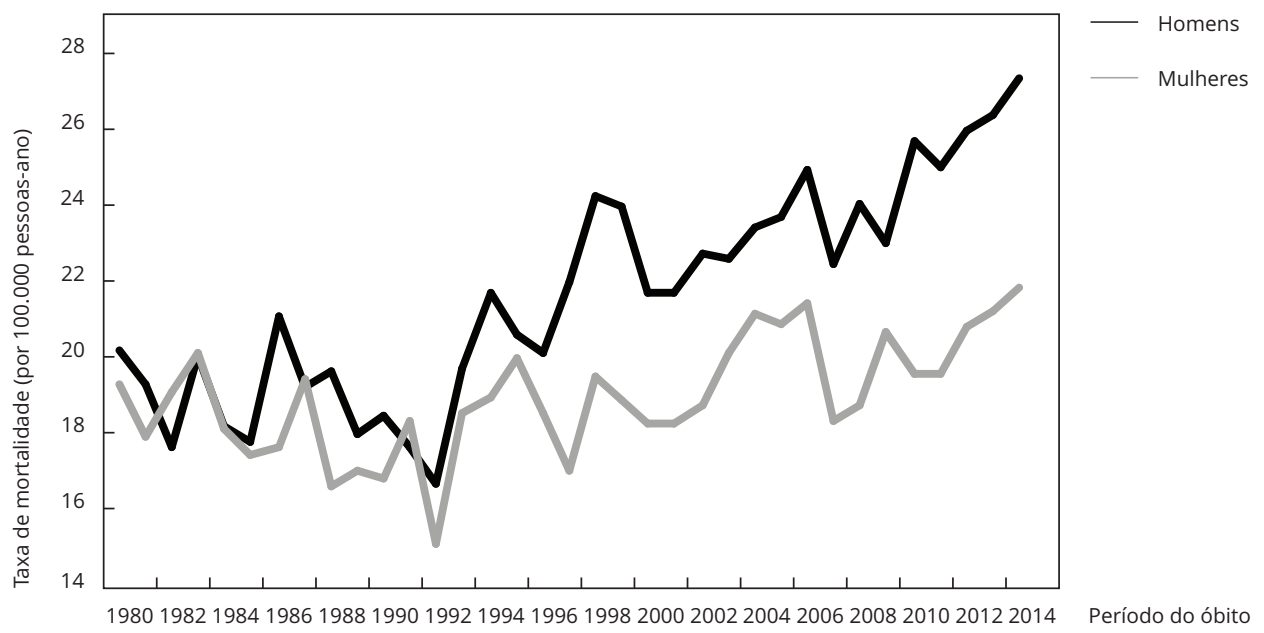

por câncer colorretal foram aproximadamente similares entre os sexos até o início da década de 1990; após os anos 1990, as taxas foram superiores no sexo masculino. Houve uma pequena redução nas taxas de mortalidade pela neoplasia no início do período, seguido por um aumento em ambos os sexos.

A Figura 2 apresenta as taxas de mortalidade distribuídas por faixa etária de acordo com sexo, período do óbito e coorte de nascimento. Encontrou-se elevação das taxas nos homens com mais de 55 anos em todo o período. Já entre as mulheres, o fenômeno ocorreu precocemente a partir dos 40 anos e de maneira acentuada até os 55 anos. Recentemente, constatou-se redução das taxas de mortalidade nos homens entre 45 e 54 anos e nas mulheres com menos de 40 anos. Considerando as coortes de nascimento, em todas as faixas etárias de ambos os sexos, foram verificadas taxas superiores nas gerações mais recentes em comparação às mais antigas.

A Figura 3 mostra um incremento progressivo nas taxas de mortalidade com o avanço da idade em ambos os sexos, tendo sido observadas as maiores taxas entre indivíduos com mais de 80 anos de idade. Em 2014, as taxas em tal grupo etário foram superiores a 172/100 mil em homens e a 141/100 mil em mulheres. Em relação à coorte de nascimento usada como referência (1935-1939), verificouse menor risco de morte pelo câncer colorretal em homens ( $R R=0,78$; IC95\%: 0,73-0,83) na coorte de 1915-1919 e o maior risco na de 1960-1964 ( $R R=1,45$; IC95\%: 1,32-1,58). Nos homens, o risco aumenta a partir da década de 1920. Já nas mulheres, o menor risco foi na coorte de 1900-1904 $(\mathrm{RR}=$ 0,95; IC95\%: 0,84-1,07) e o maior na de 1965-1969 ( $\mathrm{RR}=1,38$; IC95\%: 1,26-1,52). Nelas, o risco aumenta a partir de meados da década de 1940. Percebe-se uma leve diminuição do risco nas coortes mais recentes em ambos os sexos.

A Tabela 1 apresenta os ajustes para os modelos do efeito idade-período-coorte. Tanto o modelo idade-coorte quanto o idade-período apresentaram melhor ajuste aos dados em relação ao modelo apenas com idade e ao modelo idade-drift em ambos os sexos. O modelo completo, idade-períodocoorte, refletiu o melhor ajuste aos dados de mortalidade por câncer colorretal em ambos os sexos comparados aos modelos com apenas dois fatores, como constatado pelo menor valor de deviance. 


\section{Figura 2}

Taxa de mortalidade por câncer colorretal específica por faixa etária entre mulheres e homens de acordo com o período do óbito e a coorte de nascimento. Estado do Rio de Janeiro, Brasil, 1980 a 2014.

2a) Homens
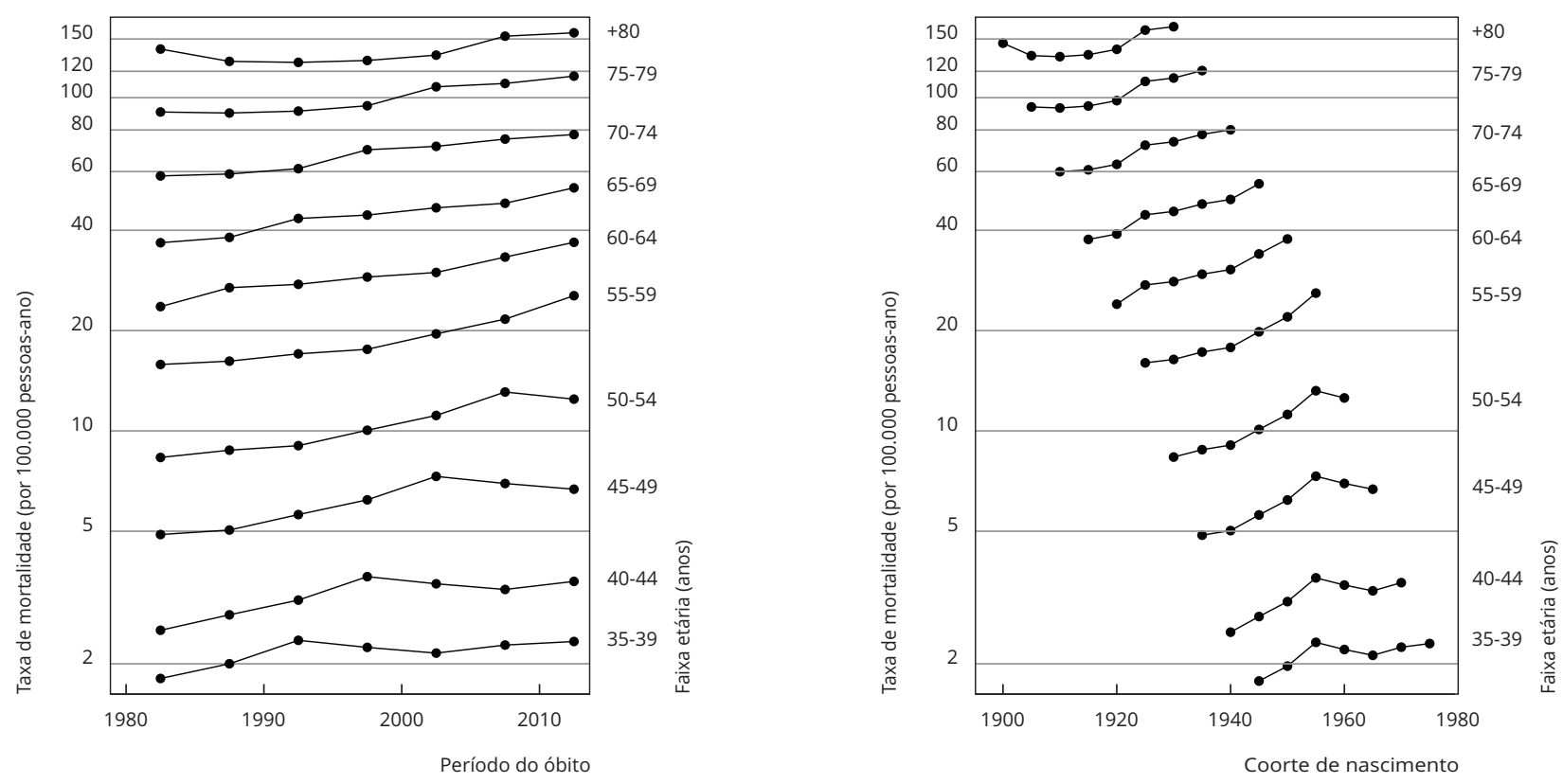

2b) Mulheres
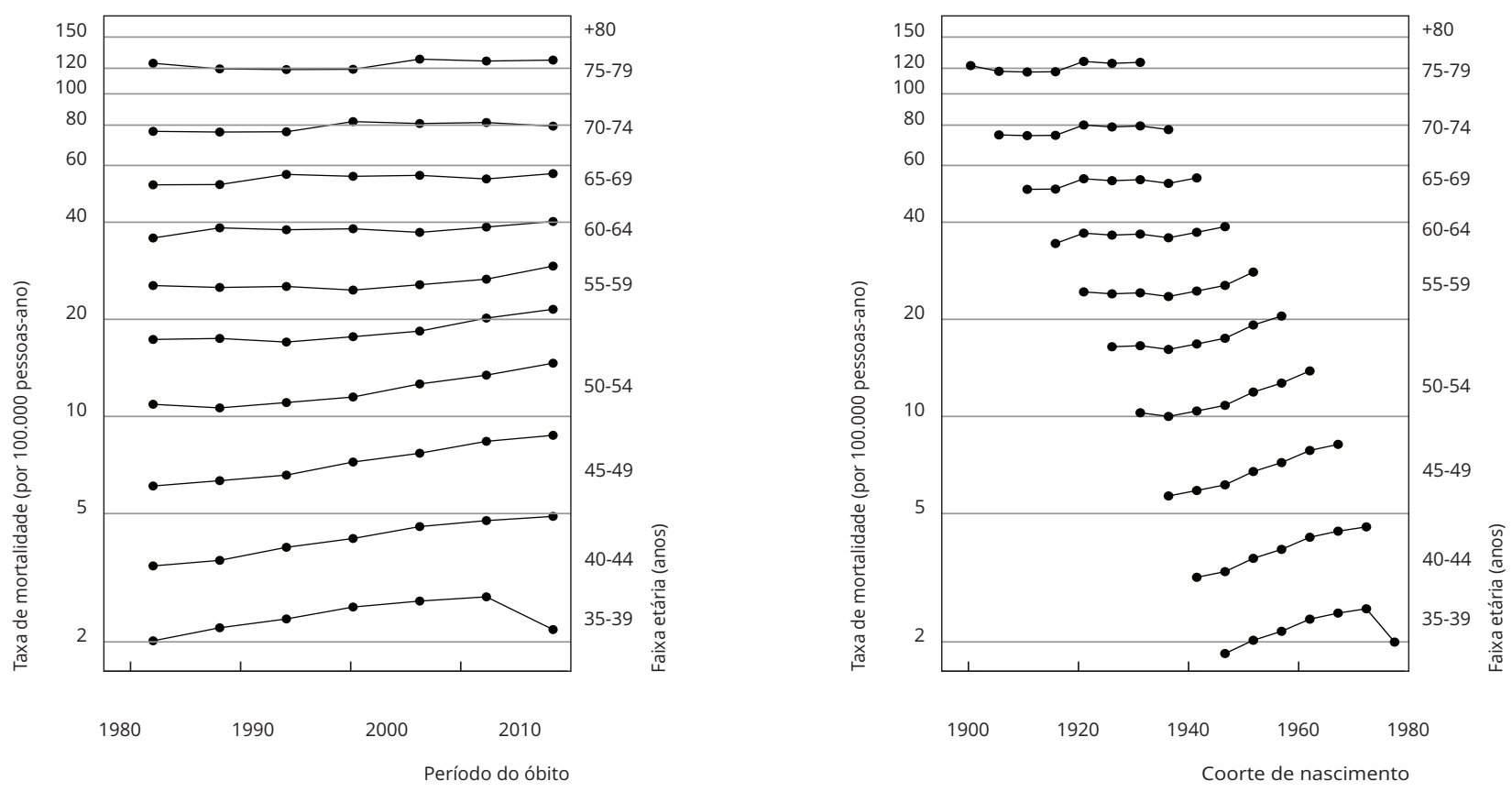
Figura 3

Estimativa dos efeitos idade-período-coorte na mortalidade por câncer colorretal. Estado do Rio de Janeiro, Brasil.

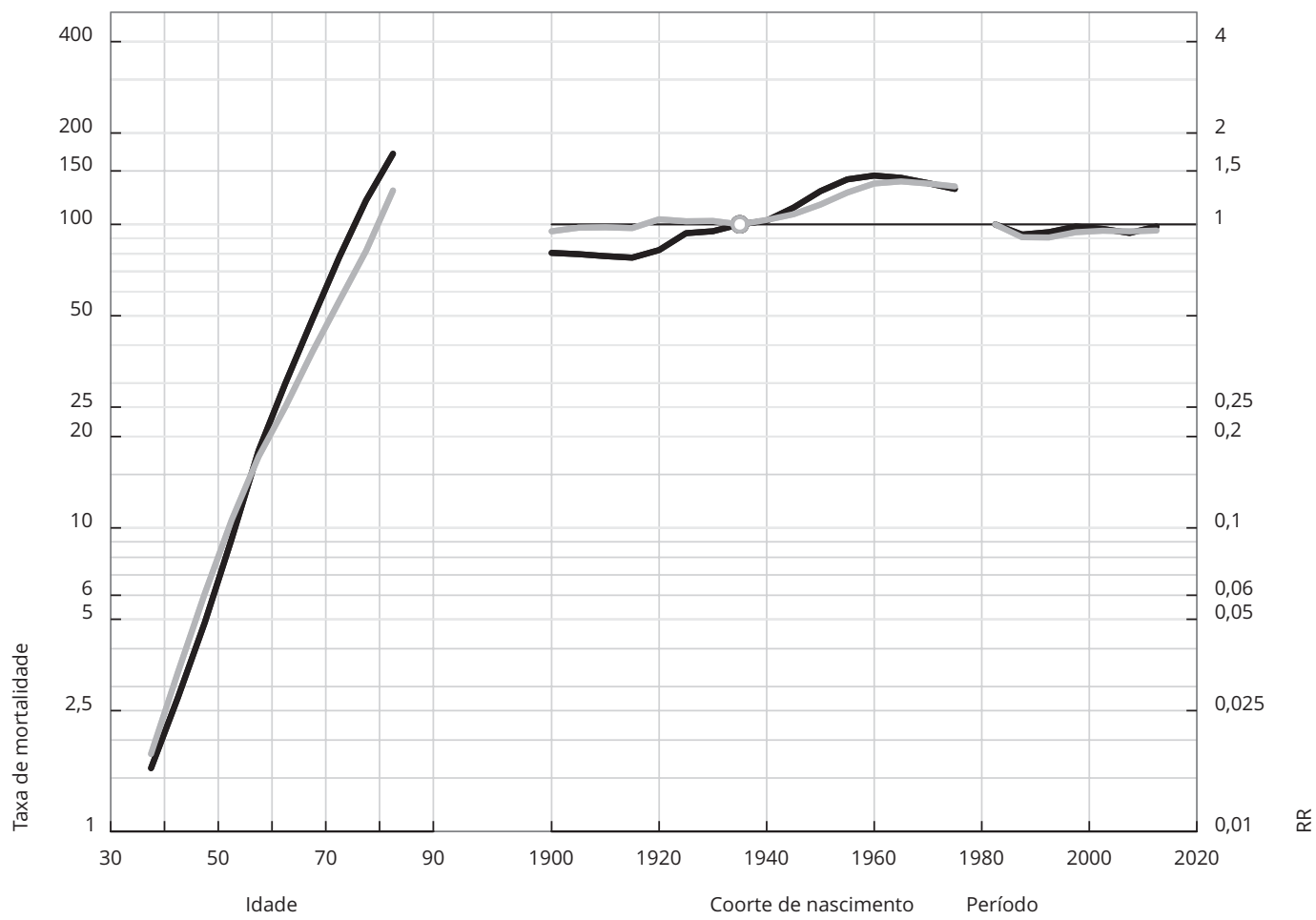

RR: risco relativo.

Nota: as curvas em preto e cinza representam, respectivamente, homens e mulheres.

\section{Tabela 1}

Ajustes para os modelos do efeito idade-período-coorte para a mortalidade por câncer colorretal de acordo com o sexo. Estado do Rio de Janeiro, Brasil, 1980 a 2014.

\begin{tabular}{|c|c|c|c|c|c|c|}
\hline \multirow[t]{2}{*}{ Modelos } & \multicolumn{3}{|c|}{ Homens } & \multicolumn{3}{|c|}{ Mulheres } \\
\hline & g.l. & Deviance & $\operatorname{Pr}(>|C h i|)$ & g.l. & Deviance & $\operatorname{Pr}(>\mid$ Chi $\mid)$ \\
\hline Idade & 62 & 327,94 & & 62 & 154,63 & \\
\hline Idade-drift & 61 & 110,56 & $<0,00001$ & 61 & 110,39 & $<0,00001$ \\
\hline Idade-coorte & 52 & 73,54 & $<0,00001$ & 52 & 67,03 & $<0,00001$ \\
\hline Idade-período-coorte & 48 & 63,52 & $<0,05$ & 48 & 55,20 & $<0,05$ \\
\hline
\end{tabular}

g.l.: grau de liberade.

\section{Discussão}

Os resultados deste estudo indicaram um aumento na mortalidade por câncer colorretal entre indivíduos de ambos os sexos com idade igual ou superior a 35 anos de idade no Estado do Rio de Janeiro entre 1980 e 2014. Tais resultados corroboram estudos anteriores que ressaltavam a elevação das taxas de mortalidade pela neoplasia ao longo do tempo no Brasil, revelando diferenças entre regiões e capitais do país 2,3,4,5,13. Nas regiões Sul e Sudeste, foram encontradas as taxas mais elevadas da 
doença 2,13. Essas diferenças regionais apontam para a contribuição de hábitos culturais e alimentares, diferenças de estilo de vida e socioeconômicas da população, além do acesso aos serviços de saúde, a qualidade do atendimento hospitalar e dos serviços de prevenção à doença 2.

A elevação da mortalidade por câncer colorretal no Rio de Janeiro confirma o padrão observado entre países em rápida transição econômica 1. Um cenário com difícil acesso à detecção e tratamento precoce da neoplasia e com limitada infraestrutura de saúde pode favorecer tal padrão. A probabilidade de sobrevivência ao câncer colorretal depende consideravelmente do estágio da doença ao diagnóstico 14 , e o tratamento é baseado em múltiplas modalidades (cirurgia, radioterapia e quimioterapia) conforme a extensão da neoplasia 15 .

O aumento das taxas de mortalidade mais pronunciado nos homens do que nas mulheres encontrado neste estudo foi previamente identificado na Espanha 16 e na República da China 17. Provavelmente, a principal explicação se deva ao fato de que as mulheres apresentam maior prevalência de uso dos serviços de saúde, em especial para a realização de exames de rotina e de prevenção, sendo essas diferenças entre os sexos mais acentuadas na idade reprodutiva 18. Além disso, mulheres tendem a prestar mais atenção aos seus processos de adoecimento que envolvem a percepção e o relato das condições de saúde 18 . Já os homens têm hábitos alimentares menos variados e elevado consumo de álcool em relação às mulheres, fato que lhes conferem maior frequência de comportamentos não saudáveis ${ }^{19}$. Por outro lado, tem sido descrito um possível efeito protetor dos hormônios femininos na tumorigênese colorretal, evidenciado com o uso de contraceptivos orais e terapia de reposição hormonal 20,21,22,23.

Neste estudo, o efeito idade na distribuição das taxas de mortalidade por câncer colorretal em ambos os sexos no período foi estatisticamente significativo. A idade é a mais importante fonte de variação nas taxas por causa do aumento do risco de morrer com o processo biológico do envelhecimento 10 e, ainda, contribui para o desenvolvimento de vários tipos de tumores 24 . Durante o processo de envelhecimento, o número de divisões celulares aumenta e podem ocorrer erros de replicação de DNA que levam às mutações. Quando essas mutações ocorrem no mecanismo de reparo do DNA, resultam no desenvolvimento de tumores 24 .

O efeito coorte verificado neste estudo revela uma elevação do risco de óbito por câncer colorretal em sucessivas gerações e uma leve diminuição do risco nas gerações mais recentes em ambos os sexos. Tal diminuição deve ser interpretada com cautela uma vez que se refere a grupos etários mais jovens, em que o número de casos da doença é menor e, portanto, merece maior cuidado a interpretação da variação aleatória inerente. Porém, pode refletir futuras tendências da doença. Resultados semelhantes aos deste estudo foram encontrados na Sérvia entre 1991 e 2010, com elevação das taxas de mortalidade no período e diminuição do risco nas coortes nascidas após a década de 196025 . O mesmo padrão foi observado na Espanha 16,26, concomitante ao aumento da incidência da doença. Por sua vez, em um estudo que avaliou a mortalidade por câncer colorretal na Europa Central, em um período de 20 anos, notou-se elevação no risco da doença em ambos os sexos em sucessivas coortes de nascimento no grupo oriental dos países (Polônia, Hungria e a então Tchecoslováquia) em contraste com os países ocidentais (Áustria, Suíça e Alemanha) onde apenas pequenas mudanças inconsistentes no risco foram encontradas 27 . Corroborando com os resultados deste estudo, um forte efeito coorte na elevação das taxas de mortalidade foi observado na República da China 17 entre 1971 e 2010. Esses achados estariam relacionados à maior exposição aos fatores de risco para o câncer colorretal e às diferenças nos hábitos alimentares da população, especificamente ao longo das gerações de nascimento.

Em contrapartida aos resultados apontados neste estudo, Inglaterra, País de Gales, Itália, Holanda, Suécia e Suíça evidenciaram efeitos de coorte de nascimento com elevação nas taxas de mortalidade nas gerações entre 1800 e 1880, com declínio significativo nas gerações subsequentes 28 . Observou-se que tal declínio precedeu a implantação do rastreamento endoscópico nesses países. Tendo em conta a similaridade entre o padrão encontrado e as doenças do aparelho digestivo superior, os autores sugeriram que alterações na tendência temporal da infecção por Helicobacter pylori poderiam afetar a tendência do câncer colorretal já que tal infecção se constitui fator de risco reconhecido para a neoplasia 28,29. Na Coreia do Sul, a diminuição do risco relativo ocorreu em gerações nascidas no início do século XX talvez influenciada por diferenças no rastreamento, estilo de vida e prevalência de infecções por coortes de nascimento ${ }^{30}$. Em Córdoba, Argentina, o mesmo padrão de diminuição do risco foi constatado apenas entre as mulheres, sendo o inverso observado nos homens 31 . 
Embora o câncer colorretal tenha tido a sua história natural inicialmente descrita na década de 1970 32, sua etiologia permanece pouco compreendida e provavelmente está relacionada a fatores genéticos e ambientais 33,34,35,36. A associação entre alimento, nutrição e risco de câncer colorretal tem sido amplamente estudada ${ }^{37}$. Existe evidência convincente de que o consumo de alimentos que contêm fibra dietética seja um fator de proteção contra a doença e de que o consumo de carne vermelha, carne processada e etanol proveniente de bebidas alcoólicas sejam fatores de risco 38. É notória a ocorrência de uma transição nutricional global que afeta particularmente os países em desenvolvimento 39. As dietas contendo alimentos processados, óleos comestíveis, bebidas açucaradas e alimentos de origem animal e as refeições sendo realizadas fora de casa convergiram para a chamada "dieta ocidental". Dietas ricas em leguminosas, outros vegetais e cereais secundários estão desaparecendo em todas as regiões e países do mundo e alguns grandes desenvolvimentos globais em tecnologia estão associados a essa mudança. Paralelamente a isso, ocorreram reduções na prática de atividade física e o aumento do sedentarismo, o que contribui para a pandemia da obesidade ${ }^{39}$, considerada um fator de risco para o câncer colorretal 40. No Brasil, constata-se um padrão de crescimento da prevalência da obesidade mais intenso entre homens do que entre mulheres, nas zonas rurais mais do que nas urbanas e entre as famílias mais pobres do que nas mais ricas 41 .

O câncer colorretal é suscetível ao rastreamento por se tratar de um grave problema de saúde com elevada incidência, morbidade e mortalidade. Possui história natural conhecida que possibilita a utilização de estratégias para a detecção precoce da neoplasia porque a maior parte dos cânceres nesta localidade evolui por conta de lesões benignas (pólipos adenomatosos) em um período pré-clínico de cerca de 10 a 15 anos 42,43. Além do mais, o tratamento é mais efetivo quando a lesão é diagnosticada precocemente ${ }^{42}$. No Brasil, a implantação de programas populacionais de rastreamento para o câncer colorretal não foi tida como viável e custo-efetiva 44. Entretanto, recomenda-se fortemente que a estratégia de diagnóstico precoce seja implementada com todos os seus componentes: ampla divulgação dos sinais de alerta para a população e profissionais de saúde, acesso imediato aos procedimentos de diagnóstico dos casos suspeitos (o que implica ampliação da oferta de serviços de endoscopia digestiva e demais suportes diagnósticos) e acesso ao tratamento adequado e oportuno 44 .

A associação entre a redução da mortalidade por essa neoplasia e a implantação de programas de rastreamento é fortemente difundida nos Estados Unidos 45, onde houve um declínio de $26 \%$ nas taxas entre 1975 e 2000. Em um modelo teórico que simulou o ocorrido, observou-se que 53\% da redução podem ser explicadas pelo rastreamento, enquanto $35 \%$ por mudanças nos fatores de risco e $12 \%$ por melhorias no tratamento 45

É necessário destacar a limitação deste estudo, inerente ao método idade-período-coorte, que é o problema de não identificabilidade. Ele surge com base na dependência linear exata entre os três fatores de interesse idade, período e coorte. Muitos autores apresentaram sugestões para a resolução do problema 6,46. Neste artigo, optou-se pelo método de ajuste de modelos com funções estimáveis proposto por Holford 11, Clayton \& Schifflers 47 que tem sido amplamente aceito e utilizado, inclusive em estudos brasileiros 48,49. Outro ponto importante é o uso da causa básica de óbito que está sujeita aos erros de classificação e, consequentemente, podem subestimar ou superestimar a magnitude das taxas de mortalidade. Embora no Brasil não tenham sido identificados estudos sobre a acurácia da informação de causa básica do óbito por câncer colorretal, outros estudos identificaram boa acurácia quando avaliados outros tipos de neoplasias no Estado do Rio de Janeiro 50,51.

Dentre as principais forças do estudo, destaca-se o uso de fonte de dados populacional. Em geral, as estatísticas de mortalidade no Brasil possuem boas cobertura e qualidade 52 . Além do mais, modelos idade-período-coorte foram desenvolvidos para quantificar a influência da idade, do período e da coorte de nascimento sobre a tendência temporal das taxas. A maioria dos estudos descreve tendências por período de óbito. Acredita-se que as tendências por coortes de nascimento têm implicações diferentes e são, consequentemente, complementares à análise por período. A exposição ao fator de risco ou fator protetor na fase inicial da carcinogênese com períodos de latência de longo prazo em diferentes gerações introduzirá um efeito de coorte. Um efeito de período pode ser atribuído a fatores de risco ou fatores de proteção envolvidos na fase tardia da carcinogênese que afetam todos os grupos etários ou a alterações nas práticas de rastreamento 53 .

Por fim, considerando o rastreamento oportunístico implantado no Brasil, as precocidades de detecção das lesões precursoras do câncer colorretal e de início do tratamento estão comprometidas 
e podem reduzir as mudanças esperadas nas taxas de mortalidade pela neoplasia e o prolongamento da expectativa de vida da população ao longo do tempo. Em contrapartida, tais fatos aliados ao envelhecimento populacional podem contribuir para um cenário ainda mais preocupante com projeções de aumento das taxas de mortalidade de câncer colorretal no país, até o ano de 202513.

\section{Conclusão}

Este estudo revelou um aumento significativo da mortalidade por câncer colorretal em homens e mulheres com 35 anos ou mais no Estado do Rio de Janeiro. Também foi observado um efeito de coorte com um aumento do risco de morte, sobretudo nas gerações mais recentes, o que pode estar ligado à adoção do estilo de vida ocidental. Tais dados enfatizam a relevância do contínuo esforço no monitoramento das taxas de incidência, mortalidade e sobrevida pela doença no país. Sem ações específicas baseadas nas evidências discutidas aqui, as taxas da doença irão aumentar nas próximas décadas. Sob o ponto de vista da saúde pública, medidas preventivas e estratégias de rastreamento visando ao diagnóstico e ao tratamento precoce de lesões precursoras devem ser implementadas no país, visto que a oferta de serviços especializados no tratamento da neoplasia é escassa e o custo que envolve o tratamento é elevado.

\section{Colaboradores}

B. Gasparini participou da concepção do estudo, coleta e análise dos dados, interpretação e apresentação dos dados, discussão e elaboração do artigo. C. M. F. P. Silva participou da concepção do estudo, interpretação e apresentação dos dados, discussão e elaboração do artigo. A. Miranda-Filho participou da interpretação, apresentação dos dados e discussão. M. Valadão participou da revisão final do artigo.

\section{Agradecimentos}

Os autores agradecem à Escola Nacional de Saúde Pública Sergio Arouca, Fundação Oswaldo Cruz (Ensp/Fiocruz). 


\section{Referências}

1. Arnold M, Sierra MS, Laversanne M, Soerjomataram I, Jemal A, Bray F. Global patterns and trends in colorectal cancer incidence and mortality. Gut 2016; 66:683-91.

2. Neves FJ, Mattos IE, Koifman RJ. Mortalidade por câncer de cólon e reto nas capitais brasileiras no período 1980-1997. Arq Gastroenterol 2005; 42:63-70.

3. Guimarães RM, Muzi CD, Boccolini CS, Boccolini PMM, Boeira SF. Tendência da mortalidade por câncer de cólon e reto no Brasil segundo sexo, 1980-2009. Cad Saúde Colet (Rio J.) 2012; 20:121-7.

4. Mendonça R, Valadão M, Couto AC, Koifman S. Tendência de mortalidade por câncer colorretal em cinco capitais brasileiras de 1980 a 2009. Cad Saúde Colet (Rio J.) 2012; 20:329-35.

5. Oliveira RC, Rêgo MAV, Oliveira RC, Rêgo MAV. Mortality risk of colorectal cancer in Brazil from 1980 to 2013. Arq Gastroenterol 2016; 53:76-83.

6. Robertson C, Gandini S, Boyle P. Age-periodcohort models: a comparative study of available methodologies. J Clin Epidemiol 1999; 52:569-83.

7. Robertson C, Boyle P. Age-period-cohort analysis of chronic disease rates. I: modelling approach. Stat Med 1998; 17:1305-23.

8. Doll R, Payne P, Waterhouse JAH, editors. Cancer incidence in five continents, v. I. Geneva: Union Internationale Contre le Cancer; 1966.

9. Carstensen B. Age-period-cohort models for the Lexis diagram. Stat Med 2007; 26:3018-45.

10. Yang Y. Trends in US adult chronic disease mortality, 1960-1999: age, period, and cohort variations. Demography 2008; 45:387-416.

11. Holford TR. The estimation of age, period and cohort effects for vital rates. Biometrics 1983; 39:311-24.

12. Clayton D, Schifflers E. Models for temporal variation in cancer rates. I: age-period and age-cohort models. Stat Med 1987; 6:449-67.

13. Souza DL, Jerez-Roig J, Cabral FJ, de Lima JRF, Rutalira MK, Costa JAG. Colorectal cancer mortality in Brazil: predictions until the year 2025 and cancer control implications. Dis Colon Rectum 2014; 57:1082-9.

14. Siegel RL, Miller KD, Fedewa SA, Ahnen DJ, Meester RGS, Barzi A, et al. Colorectal cancer statistics, 2017. CA Cancer J Clin 2017; 67:177-93.

15. National Comprehensive Cancer Network. NCCN clinical practice guidelines in oncology. https://www.nccn.org/professionals/ physician_gls/f_guidelines.asp (acessado em 08/Ago/2017).

16. Ribes J, Navarro M, Cleries R, Esteban L, Pareja L, Binefa G, et al. Colorectal cancer mortality in Spain: trends and projections for 1985-2019. Eur J Gastroenterol Hepatol 2009; 21:92-100.
17. Su S-Y, Huang J-Y, Jian Z-H, Ho C-C, Lung C-C, Liaw Y-P. Mortality of colorectal cancer in Taiwan, 1971-2010: temporal changes and age-period-cohort analysis. Int J Colorectal Dis 2012; 27:1665-72.

18. Pinheiro RS, Viacava F, Travassos C, Brito AS. Gênero, morbidade, acesso e utilização de serviços de saúde no Brasil. Ciênc Saúde Coletiva 2002; 7:687-707.

19. Svensson E, Grotmol T, Hoff G, Langmark F, Norstein J, Tretli S. Trends in colorectal cancer incidence in Norway by gender and anatomic site: an age-period-cohort analysis. Eur J Cancer Prev 2002; 11:489-95.

20. dos Santos Silva I, Swerdlow AJ. Sex differences in time trends of colorectal cancer in England and Wales: the possible effect of female hormonal factors. Br J Cancer 1996; 73:692-7.

21. Fernandez E, Bosetti C, La Vecchia C, Levi F, Fioretti F, Negri E. Sex differences in colorectal cancer mortality in Europe, 1955-1996. Eur J Cancer Prev 2000; 9:99-104.

22. La Vecchia C, Gallus S, Fernandez E. Hormone replacement therapy and colorectal cancer: an update. Br Menopause Soc J 2005; 11:166-72.

23. Majek O, Gondos A, Jansen L, Emrich K, Holleczek B, Katalinic A, et al. Sex differences in colorectal cancer survival: population-based analysis of 164,996 colorectal cancer patients in Germany. PLoS One 2013; 8:e68077.

24. Richardson B. Impact of aging on DNA methylation. Ageing Res Rev 2003; 2:245-61.

25. Ilic M, Ilic I. Colorectal cancer mortality trends in Serbia during 1991-2010: an age-period-cohort analysis and a joinpoint regression analysis. Chin J Cancer 2016; 35:55.

26. Lopez-Abente G, Pollan M, Vergara A, Moreno C, Moreo P, Ardanaz E, et al. Age-period-cohort modeling of colorectal cancer incidence and mortality in Spain. Cancer Epidemiol Prev Biomark 1997; 6:999-1005.

27. Evstifeeva TV, Macfarlane GJ, Robertson C. Trends in cancer mortality in central European countries: the effect of age, birth control and time-period. Eur J Public Health 1997; 7:169-76.

28. Sonnenberg A. Effects of birth cohort on long-term trends in mortality from colorectal cancer. Clin Gastroenterol Hepatol 2012; 10:1389-94.

29. Zumkeller N, Brenner H, Zwahlen M, Rothenbacher D. Helicobacter pylori infection and colorectal cancer risk: a meta-analysis. Helicobacter 2006; 11:75-80.

30. Jee Y, Oh C-M, Shin A. Recent decrease in colorectal cancer mortality rate is affected by birth cohort in Korea. Asian Pac J Cancer Prev 2015; 16:3951-5.

31. Pou SA, Osella AR, Eynard AR, Niclis C, del Pilar Diaz M. Colorectal cancer mortality trends in Córdoba, Argentina. Cancer Epidemiol 2009; 33:406-12. 
32. Hill MJ, Morson BC, Bussey HJ. Aetiology of adenoma: carcinoma sequence in large bowel. Lancet 1978; 1:245-7.

33. Hamilton SR, Aaltonen LA, editors. World Health Organization classification of tumours. Pathology and genetics of tumours of the digestive system. Lyon: IARC Press; 2000.

34. Fearon ER, Vogelstein B. A genetic model for colorectal tumorigenesis. Cell 1990; 61:759-67.

35. Potter JD. Colorectal cancer: molecules and populations. J Natl Cancer Inst 1999; 91:916-32.

36. Cutait R, Cotti G, Garicochea B, Muraro C, Carvalho F, Leite K, et al. Instabilidade microssatélites em portadores de câncer colo-retal. Revista Brasileira de Coloproctologia 2002; 4:225-323.

37. Norat T, Chan D, Lau R, Aune D, Vieira R. The associations between food nutrition and physical activity and the risk of colorectal cancer. London: Imperial College London; 2010. (WCRF/AICR Systematic Literature Review Continuous Update Project Report).

38. World Cancer Research Fund. Colorectal Cancer 2011 Report: food, nutrition, physical activity, and the prevention of colorectal cancer. Washington DC: American Institute for Cancer Research; 2012.

39. Popkin BM, Adair LS, Ng SW. Global nutrition transition and the pandemic of obesity in developing countries. Nutr Rev 2012; 70:3-21.

40. Renehan AG, Tyson M, Egger M, Heller RF, Zwahlen M. Body-mass index and incidence of cancer: a systematic review and meta-analysis of prospective observational studies. Lancet 2008; 371:569-78.

41. Monteiro CA, D’A Benicio MH, Conde WL, Popkin BM. Shifting obesity trends in Brazil. Eur J Clin Nutr 2000; 54:342-6.

42. Instituto Nacional de Câncer; Sociedade Brasileira de Colproctologia; Colégio Brasileiro de Cirurgiões; Associação Brasileira de Colite Ulcerativa e Doença de Crohn; Colégio Brasileiro de Cirurgia Digestiva; Sociedade Brasileira de Endoscopia Digestiva; et al. Falando sobre o câncer de intestino. Rio de Janeiro: Instituto Nacional de Câncer; 2003.

43. Tarraga López PJ, Albero JS, Rodríguez-Montes JA. Primary and secondary prevention of colorectal cancer. Clin Med Insights Gastroenterol 2014; 7:33-46.
44. Departamento de Atenção Básica, Secretaria de Atenção à Saúde, Ministério da Saúde. Rastreamento, 2010. Brasil. Brasília: Ministério da Saúde; 2010.

45. Edwards BK, Ward E, Kohler BA, Eheman C, Zauber AG, Anderson RN, et al. Annual report to the nation on the status of cancer, 19752006, featuring colorectal cancer trends and impact of interventions (risk factors, screening, and treatment) to reduce future rates. Cancer 2010; 116:544-73.

46. O’Brien RM. Age-period-cohort models: approaches and analyses with aggregate data. Boca Raton: CRC Press; 2015.

47. Clayton D, Schifflers E. Models for temporal variation in cancer rates. II: age-period-cohort models. Stat Med 1987; 6:469-81.

48. Borges AKM, Miranda-Filho A, Koifman S, Koifman RJ. Thyroid cancer incidences from selected South America population-based cancer registries: an age-period-cohort study. J Glob Oncol 2017; 4:1-11.

49. Miranda-Filho AL, Koifman RJ, Koifman S, Monteiro GTR. Brain cancer mortality in an agricultural and a metropolitan region of Rio de Janeiro, Brazil: a population-based, ageperiod-cohort study, 1996-2010. BMC Cancer 2014; 14:320.

50. Queiroz RCS, Mattos IE, Monteiro GTR, Koifman S. Reliability and accuracy of oral cancer as the reported underlying cause of death in the Municipality of Rio de Janeiro. Cad Saúde Pública 2003; 19:1645-53.

51. Miranda-Filho AL. Mortalidade por neoplasias potencialmente associadas à atividade agrícola no estado do Rio de Janeiro. Rio de Janeiro: Escola Nacional de Saúde Pública Sergio Arouca, Fundação Oswaldo Cruz; 2012.

52. França E, de Abreu DX, Rao C, Lopez AD. Evaluation of cause-of-death statistics for Brazil, 2002-2004. Int J Epidemiol 2008; 37:891901.

53. Chauvenet M, Cottet V, Lepage C, Jooste V, Faivre J, Bouvier A-M. Trends in colorectal cancer incidence: a period and birth-cohort analysis in a well-defined French population. BMC Cancer 2011; 11:282. 


\section{Abstract}

The aim of this study was to estimate the contribution of the effect of age, period, and birth cohort on mortality from colorectal cancer. The study analyzed data on deaths from this cancer in individuals over 35 years of age in Rio de Janeiro State, Brazil, obtained from the Mortality Information System (SIM) from 1980 to 2014. Mortality rates were calculated by gender and age bracket. The effect of age, period, and birth cohort was estimated by the method that uses estimable functions: deviations, curves, and drift in the Epi library of the $R$ software. Standardized mortality rates from colorectal cancer were 27.37/100,000 men and 21.83/100,000 women in 2014. The data showed an increase in mortality from this cancer from 1980 to 2014, and mortality rates were higher in men than in women after the 1990s. Age effect was observed with an increase in the rates and aging. Generational analysis showed lower risk of death in older versus younger cohorts, possibly related to the adoption of the Western lifestyle. This scenario underscores the need for screening strategies aimed at early diagnosis and treatment of precursor lesions.

Colorectal Neoplasms; Age Effect; Period Effect; Cohort Effect

\section{Resumen}

El objetivo de este trabajo fue estimar la contribución del efecto de la edad, del periodo y de la cohorte de nacimiento en la mortalidad por cáncer colorrectal. Se analizaron datos de óbitos por la neoplasia entre individuos con más de 35 años de edad del estado de Río de Janeiro, Brasil, extraídos del Sistema de Informaciones sobre Mortalidad (SIM) entre 1980 y 2014. Las tasas de mortalidad fueron calculadas por género y franja de edad. El efecto de la edad, período y cohorte de nacimiento fue estimada por el método que utiliza funciones estimables: desvios, curvaturas y drift, en la biblioteca Epi del software R. Las tasas de mortalidad por cáncer colorrectal estandarizadas fueron 27,37/100 mil hombres y 21,83/100 mil mujeres en 2014. Se verificó un aumento de la mortalidad por la neoplasia entre 1980 y 2014, siendo las tasas de mortalidad entre hombres superiores a las de las mujeres tras la década de 1990. El efecto edad se observó con el aumento de las tasas y el envejecimiento. El análisis de las generaciones mostró un menor riesgo de óbito en las cohortes más antiguas, comparadas con las cohortes más recientes, lo que puede estar vinculado a la adopción del estilo de vida occidental. Tal escenario apunta la relevancia de la implantación de estrategias de monitoreo con el objetivo del diagnóstico y el tratamiento precoz de lesiones precursoras de la enfermedad.

Neoplasias Colorrectales; Efecto Edad; Efecto Periodo; Efecto de Cohortes

Recebido em 05/Mar/2017

Versão final reapresentada em 12/Set/2017

Aprovado em 09/Out/2017 